ISSN 1112-9867

http://www.jfas.info

\title{
HYDROTHERMAL COMPLEX OF THE SOUK AHRAS BASIN: GEOLOGICAL AND HYDROGEOCHEMICAL APPROACHES (NORTH EAST OF ALGERIA)
}

\author{
Y. Bouroubi-Ouadfel ${ }^{1,2}$, M. Djebbar ${ }^{2}$ and A. Khiari $^{1}$ \\ ${ }^{1}$ Faculty of Earth Sciences and architecture, RNAMS Laboratory, Larbi Ben M'hidiUniversity \\ Oum El Bouaghi, Algeria \\ ${ }^{2}$ Department of Geological Sciences. Mentouri 1 University, Ain El Bey, 25010 Constantine,
} Algeria

Received: 17 May 2016 / Accepted: 26 August 2016 / Published online: 01 September 2016

\begin{abstract}
North- East of Algeria, in The Souk Ahras region, the Triassic evaporates are in the form of important intrusive masses. Thermal and cold water emerge from various training. These sources present are taking their pathways along the faulting system. A complex multilayered reservoir has significant potential water. The karstic aquifer consists mainly on fresh water. Thermal water characterized by high salinity is carbo-gaseous. Collection and chemical analysis of major water elements in addition to nonionic mineral compounds $\left(\mathrm{SiO}_{2}\right)$ and trace elements $\left(\mathrm{Sr}^{2+}, \mathrm{F}^{-}, \mathrm{Br}^{-}\right)$have determined a deep saline fluid circulation. The tectonic effect would be responsible for the current water flow. Cartography of fracturing system has identified a NNW-SSE hot spring distribution. Similar alignment can match the faulting system direction affecting the concerned study area.
\end{abstract}

Keywords: Triassic evaporate; thermal waters; tectonic; deep fluid circulation.

Author Correspondence, e-mail: minabouroubi.oeb@gmail.com

doi: http://dx.doi.org/10.4314/jfas.v8i3.13 


\section{INTRODUCTION}

Le Nord-est Algérien d'une aire de plus de $70000 \mathrm{~km}^{2}$, s'étend jusqu'à 200km au Sud de la mer méditerranée, depuis Bejaia à la frontière Tunisienne. Les formations carbonatées, généralement karstifiées, affleurent sur une superficie de $7200 \mathrm{~km}^{2}$ [1], elles constituent plusieurs unités hydrogéologiques [2], et renferment un important potentiel en eau, les plus sollicitées sont les Karsts de : Rémila- Khenchela [3]; Hamma-Bouziane [4] ; Taoura [5] ; Sud Sétifien [6]. La majorité de ces aquifères karstiques renferment des drains d'eau chaude. En effet, le plus grand nombre de sources thermales du Nord de l'Algérie se trouvent dans l'Est algérien [7] et [8], les réservoirs géothermiques sont complexes et discontinus [7]. Afin de déterminer l'origine de la minéralisation et les modes de circulations des eaux dans le complexe aquifère de la région de Souk Ahras, la géochimie et l'étude de la fracturation ont étés utilisés; des prélèvements d'eaux de différents aquifères ont été analysés en périodes de haute et basse eaux et les principales failles ont été cartographiées.

La région à l'étude appartient au système hydrothermal dans la terminaison Nord-est du domaine atlasique et au contact du domaine tellien charrié [9] dans la région des diapirs.

\subsection{Cadre géographique}

Constituant la partie septentrionale de la zone des diapirs, le secteur d'étude de superficie de plus de $1700 \mathrm{~km}^{2}$, se trouve à $80 \mathrm{~km}$ au sud de la mer méditerranée, aux confins Algéro-Tunisiens (Fig.1). Cette région appartient à la zone de transition entre l'atlas tellien et l'atlas saharien. Le climat est subhumide dans la partie Nord et nettement semi-aride à aride dans sa partie méridionale, il est caractérisé par des précipitations irrégulières (en moyenne $550 \mathrm{~mm} \cdot \mathrm{an}^{-1}$ ) [10], la température moyenne de l'air est prés de $15^{\circ} \mathrm{C}$ avec un fort pouvoir évaporant (74\%). Deux oueds caractérisent la zone; Medjerda au Nord et Mellegue au Sud, ils se poursuivent jusqu'en Tunisie pour constituer une seul rivière qui débouche enfin en mer méditerranée [11]. La couverture forestière est très dense au Nord sur les massifs septentrionaux appelée : monts de la Medjerda et fortement dégradée au fur et à mesure qu'on progresse vers le Sud. 


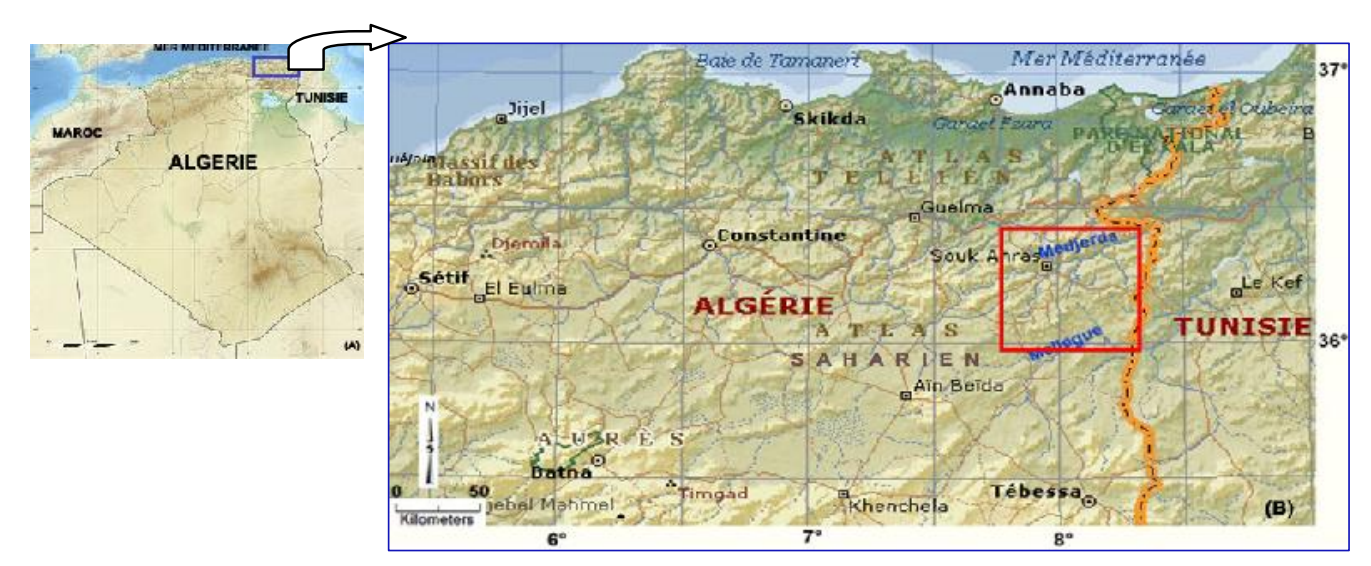

Fig. 1. (A) Localisation de l'Algérie dans le bassin méditerranéen, le rectangle bleu représente le Nord-est. (B) Le rectangle rouge montre la région d'étude.

\subsection{Cadre géologique}

La région de Souk Ahras se situe dans la bordure septentrionale atlasique et au contact du domaine tellien charrié de la chaîne des maghrébides [12]. L'atlas saharien est caractérisé par d'épaisses formations mésozoïques (environ 2500m) [13] plissées et fracturées, présentant la particularité d'une lacune stratigraphique du Jurassique, et d'importantes masses triasiques en affleurement disposées parallèlement à la chaîne, l'atlas tellien est formé d'un empilement de nappes de charriages de différents domaines paléogéographiques [9]. En confins algéro-tunisien, affleurent les unités du Flysch numidien et les formations du domaine externe de la chaîne des Maghrébides. Les unités du Flysch numidien constituent d'épaisses séries gréseuses disposées en contact anormal sur les séries allochtones telliennes et parautochtones de l'avant pays atlasique [9]. La région sud appartient à une large zone synclinoriale subsidente qui s'étend d'Aïn Beïda à Merahna aux confins Algéro- tunisiens (Fig. 1 et 2). La partie Nord constitue le flanc sud des monts de la Medjerda [13]. Les formations de l'autochtone sont: le Trias, composé d'évaporites à fragments et blocs de roches diverses; les formations crétacées $(2500 \mathrm{~m})$ sont à dominance carbonatée intercalée de marnes ; le Paléogène est essentiellement marneux à rares passées calcaires (200m) ; les sédiments néogènes d'épaisseurs variables sont très hétérogènes mais principalement de type marin, le miocène continental est constitué de poudingues, argiles rouges et grises, calcaires lacustres, il occupe le cœur du fossé d'effondrement de Taoura. Le Quartenaire est 
formé de graviers, sables, limons, brèches, travertins.
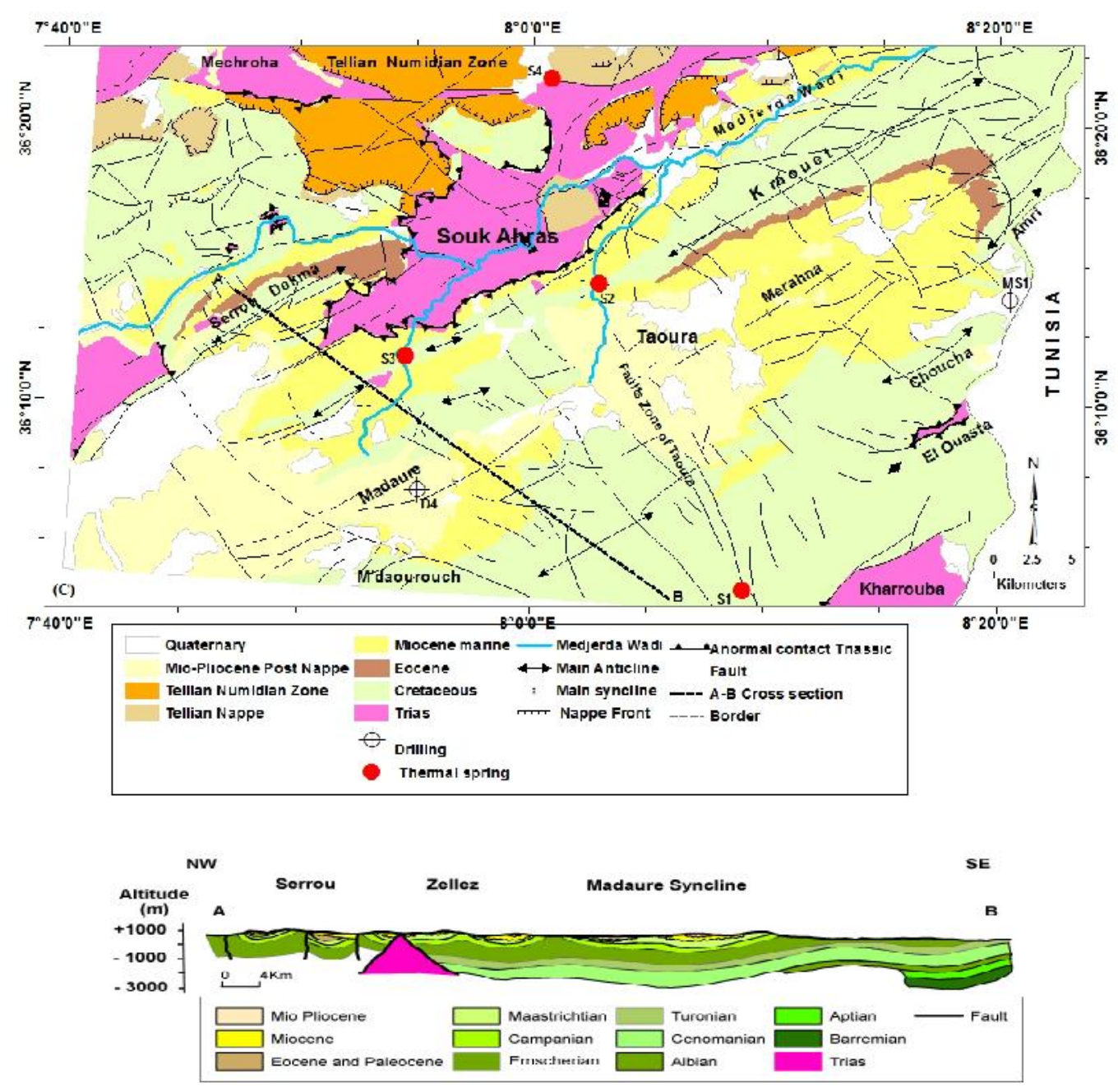

Fig.2. (C) Carte géologique et structurale simplifié de la région d'étude avec localisation des points d'eaux utilisés dans cette étude. (D) Coupe géologique.

\subsection{Cadre hydrogéologique}

Le complexe aquifère de Souk Ahras est un système multicouche [14]. Les principaux réservoirs hydrogéologiques sont:

L'aquifère miocène marin: Le Forage (D4) capte l'aquifère des grès miocènes (0 à 95m) au de là, il n'y a pas eu de remontée de cuttings, il était arrêté à (146m) [15].

L'aquifère de l'éocène autochtone, présente quelques sources froides $\left(<02 \mathrm{~L}^{-1} \mathrm{~s}^{-1}\right)$ à haute altitude, elles sont directement influencées par les eaux des précipitations et de la neige. L'aquifère du karst Maastrichtien-Campanien: présente un aquifère très développé dans 
les calcaires crayeux. Il met à jour plusieurs sources avec un débit $\left(<15 \mathrm{~L}\right.$. $\left.\mathrm{s}^{-1}\right)$ [16], cet aquifère est exploité par forages atteignant une profondeur entre (150 à 350m).

Les aquifères du Turonien et l'Aptien: au Sud et Sud- Est de Taoura, une dizaine de forages de reconnaissances hydrogéologiques furent abandonné à cause de la salinité des eaux $(\mathrm{EC}=$ $7.2 \mathrm{~ms} / \mathrm{cm}$ ). Ils atteignent les aquifères du Turonien et surtout de l'Aptien vers une profondeur $(<250 \mathrm{~m})$. Dans la partie méridionale du bassin d'étude, ces formations carbonatées affleurent en surface. En 1996, des sondages réalisés par l'Office de recherche géologique et minière- Algérie dans la région de El Ouasta (Fig. 2) ont recoupé ces formations (327.4 $562.1 \mathrm{~m})$. Certains intervalles montrent une minéralisation polymétallique liée à des petites fissures, caractérisée par la sphalérite $(\mathrm{ZnS})$, galène $(\mathrm{PbS})$, chalcopyrite $\left(\mathrm{CuFeS}_{2}\right)$, cuivre gris, pyrite $\left(\mathrm{FeS}_{2}\right)$, barytine $\left(\mathrm{BaSO}_{4}\right)$ et calcite $\left(\mathrm{CaCO}_{3}\right)$. Ces minéraux sont souvent associés à des petites veinules remplies de bitume [17] Le contact entre Aptien et Trias se caractérise par zone ferro-barytique à Célestine.

L'aquifère profond: met à jour de sources thermales qui sourdent toutes à travers des accidents profonds affectant différentes formations géologiques. (S1, S2 et S4), se caractérisent par une odeur de soufre très marquée.

Dans la partie septentrionale de la zone des diapirs se trouvent quatre principales sources thermales, chacune d'elles présentent au moins trois griffons.

Notons que l'aquifère du Miocène gréseux et l'aquifère karstique maastrichtien- campanien sont exploités pour l'alimentation en eau potable, l'irrigation des terres culturales et quelques besoins industriels. Tous les forages productifs traversent des zones très karstifiées. (Au cours de leurs réalisations, des pertes totales de boue de forage ont étés signalées).

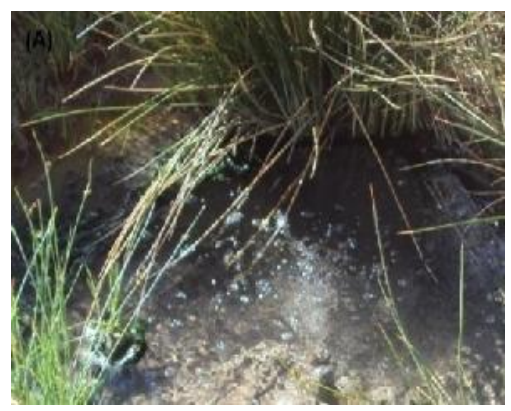

Fig.3. (A) Bulles de gaz.

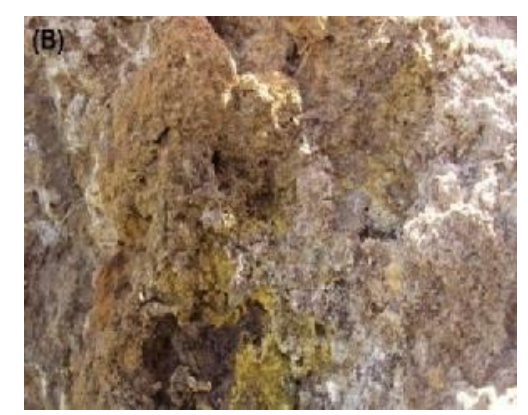

(B) Soufre près du grifón principal de El Demssa.

(Photos: Bouroubi Y. Septembre 2014). 
Tableau 1. Caractéristiques physiques des points d'eau et conditions d'émergence des sources thermals

\begin{tabular}{|c|c|c|c|c|}
\hline $\begin{array}{l}\text { Nom du } \\
\text { point }\end{array}$ & $\begin{array}{l}\text { Débit } \\
\text { L. } s^{-1}\end{array}$ & $\begin{array}{c}\text { Température } \\
{ }^{\circ} \mathrm{C}\end{array}$ & $\begin{array}{c}\mathrm{CE} \\
(\mathrm{ms} / \mathrm{cm})\end{array}$ & Conditions d'émergence \\
\hline$* \mathrm{El}$ & 2 & $26 \pm 1$ & 6.6 & Faille affectant les calcaires $\mathrm{du}$ \\
\hline $\begin{array}{l}\text { Demssa } \\
\text { (S1) }\end{array}$ & & & & Coniacien. \\
\hline $\begin{array}{l}\text { H. Tassa } \\
\quad(\mathrm{S} 2)\end{array}$ & 3.8 à 4 & $40 \pm 0.5$ & 3.52 & $\begin{array}{l}\text { Faille entre calcaires Maastrichtiens } \\
\text { et grès du Miocène. }\end{array}$ \\
\hline $\begin{array}{c}\text { El } \\
\text { Khengua } \\
\text { (S3) }\end{array}$ & 8 à 16 & $26.5 \pm 0.5$ & 1.65 & $\begin{array}{l}\text { Faille entre calcaires Maastrichtiens } \\
\text { et conglomérats du Miocène. }\end{array}$ \\
\hline $\begin{array}{l}\text { Ouled Zaid } \\
\qquad(\mathrm{S} 4)\end{array}$ & 6 à 8 & $39 \pm 0.5$ & 2.04 & $\begin{array}{l}\text { Accident entre Trias et calcaire de } \\
\text { l'Eocène Allochtone. }\end{array}$ \\
\hline $\mathrm{D} / \mathrm{D} 4$ & Pompé à 100 & $20.8 \pm 0.2$ & 1.3 & $\begin{array}{l}\text { Forage Karst et grès miocène } \\
(146 \mathrm{~m}) \text {. }\end{array}$ \\
\hline D/MS1 & Pompé à 25 & 17.4 & 1 & Forage Karst (264m). \\
\hline
\end{tabular}

* Ces griffons sont méconnus ou abandonnés faute de chemins d'accès. Ils n'existent pas dans la bibliographie avant nos travaux.

\section{MATÉRIEL ET MÉTHODES}

Des prélèvements ont été effectués au niveau de six points d'eau (sources, forages) en deux périodes Mars- 2012 et septembre- 2014 pour analyses des éléments majeures, traces. La température, le potentiel d'Hydrogène et la conductivité électrique ont été mesurés in situ. Au moment des prélèvements, les forages étaient déjà en pompage. La température a été prise à l'exutoire des sources et des forages au moyen d'une sonde de température intégrée au pH-mètre portatif de marque Hanna (HI 9025). La valeur de température est donnée en ${ }^{\circ} \mathrm{C}$. L'incertitude de la mesure de température est de $\pm 0.2^{\circ} \mathrm{C}$. La mesure du $\mathrm{pH}$ de l'eau est réalisée à l'aide d'une électrode pH reliée au pH-mètre de marque Hanna (HI 9025). La mesure de la conductivité électrique, a été réalisée à l'aide d'une électrode reliée au conductimètre portatif de marque Hanna (HI 9033). Les éléments majeurs analysés sont (Ca, $\mathrm{Mg}, \mathrm{Na}, \mathrm{K}, \mathrm{HCO}_{3}, \mathrm{Cl}, \mathrm{SO}_{4}, \mathrm{NO}_{3}$ ), traces ( $\mathrm{Sr}, \mathrm{F}, \mathrm{Br}$ ) et la $\mathrm{SiO}_{2}$. Les ions calcium et magnésium ont été analysés par spectrométrie à flux continu auto-analyseur. Le sodium et le potassium ont été dosés par photométrie de flamme. Les bicarbonates ont été analysés par 
volumétrie, les sulfates par dosage turbidimétrique, et les chlorures ainsi que les nitrates ont été dosés par colorimétrie auto-analyseur à flux continu. Les analyses en éléments majeurs on étés réalisées au niveau du Laboratoire régional de l'ANRH de Constantine. La précision des mesures est de l'ordre de $\pm 5 \%$. La Silice et le fluor ont étés analysés par colorimétrie auto-analyseur au niveau du Laboratoire de l'ANRH- Alger. L'analyse du strontium a été analysée en deux laboratoires différents: celle de mars -2012 au CNRA (Centre de Recherche Nucléaire d'Alger) par ICP-MS_Jobin Yvon 32 et celle de septembre- 2014 au niveau du laboratoire GIS de Nîmes par ICP -MS. Le brome a été analysé par chromatographie à GIS. L'eau prélevée de chaque point pour ces analyses a été filtrée avec un filtre de $0.45 \mu \mathrm{m}$ et acidifiée avec l'acide Nitrique concentrée à $65 \%$ sur terrain jusqu'à $\mathrm{pH}=2$ et conservée dans des flacons en polyéthylène de $250 \mathrm{ml}$.

Les variables de la pression partielle équilibrante de dioxyde de carbone et les indices de saturation vis-à-vis de la calcite, de la dolomite, du gypse et d'autres minéraux ont été calculées à l'aide du logiciel «Phreeqci-3.1.4-8929».

Une analyse de la fracturation du bassin d'étude a été effectuée sur l'assemblage des quatre cartes géologiques au 1/50 000 de Souk Ahras, Oued Mougras, M'Daourouch et Taoura après numérisation et géo référencement (système de projection WGS 1984 UTM 32N) avec Arc Gis 10.1.

\section{RESULTAS ET DISCUSSION}

\subsection{Analyse de la fracturation}

L'examen structural à partir des cartes géologiques, (Erreur ! Source du renvoi introuvable.. 2) appuyé de données de géophysiques [15] et les coupes de forages met en évidence une disposition en blocs des formations crétacées délimitées par des accidents diagonaux décrochant dextres NW-SE, (la transversale de M'daourouch et celle qui limite le graben de Taoura) et senestres NE-SW auxquelles sont associées des failles E-W. Suite à la distension et compression du domaine Atlasique accordée par tous les auteurs [13,18, 7, 19] des horsts et grabens se sont mis en place, le fossé d'effondrement de Taoura est spectaculaire.

L'analyse da la carte (Fig. 4) montre une disposition NNW-SSE des sources thermales de la 
partie septentrionale de la zone des diapirs.

En Algérie, la tectonique active est localisée dans le tell et l'atlas saharien; conséquence de la convergence continue des plaques Afrique et Eurasie [20]. L'activité tectonique de l'Algérie orientale a donné lieu à un flux de chaleur élevé supérieure à la normale [7] avec un gradient géothermique de $5^{\circ} \mathrm{C} / 100 \mathrm{~m}$. Les diapirs du Trias sont issus de failles profondes [21].

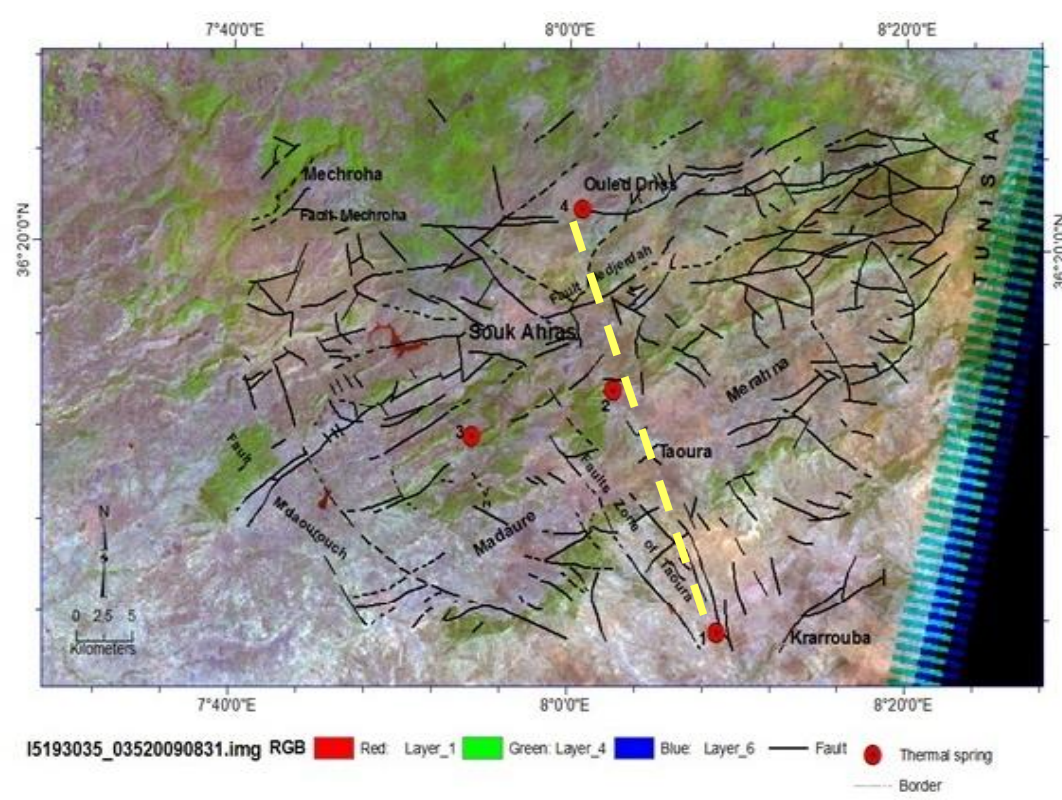

Fig. 4. Principales failles de la région de Souk Ahras sur image satellite montrant la disposition NNW SSE des sources thermales.

\subsection{Paramètres physiques et éléments majeurs}

Pour le Nord de l'Algérie, [22] considère, d'après l'examen des températures atmosphériques, que les eaux des sources qui ont une température $\left(\geq 22^{\circ} \mathrm{C}\right)$ sont thermales, ainsi, trois groupes sont identifiés: Groupe (I) eaux Ortho- thermales $\left(37^{\circ} \mathrm{C} \leq \mathrm{T}^{\circ} \mathrm{C} \leq 40^{\circ} \mathrm{C}\right)$, inclus les sources (S2) et (S4). Groupe (II) eaux Méso-thermales $\left(22^{\circ} \mathrm{C} \leq \mathrm{T}^{\circ} \mathrm{C} \leq 37^{\circ} \mathrm{C}\right)$, inclus les sources (S1) et (S3). Groupe (III) correspond aux eaux froides des aquifères karstiques du Crétacé supérieur, et grès miocène. La température joue un rôle important dans la solubilité des sels dissous et sur l'activité chimique. La minéralisation totale dissoute entre $(1 \mathrm{~g} / \mathrm{L}$ à $5.2 \mathrm{~g} / \mathrm{L}$ ) indique le caractère saumâtre de la majorité des eaux mises à part celles issues des eaux des précipitations qui alimentent les niveaux superficiels du karst représentées ici par (MS1 avec 0.6g/L). Le pH indique des eaux légèrement acides. 
La classification de Stabler (Table 2) désigne deux grands types d'eaux, Bicarbonatées et chlorurées sodiques des groupes (I et II) et Bicarbonatées calciques concernant les eaux froides. Les bonnes corrélations des bicarbonates et les chlorures avec la conductivité électrique (Fig.5. a et b) montrent la détermination de ces deux substances dans la minéralisation totale des eaux. Ce sont des eaux carbo-gazeuses et saumâtres. La présence de fortes teneurs de chlorures peut être liée à seulement un phénomène ou à la combinaison de plusieurs processus distincts $[23,24]$. Compte tenu de la distance par rapport à la mer et le contexte géologique, deux origines possibles peuvent expliquer ces teneurs ; dissolution de minéraux évaporitiques et/ou contribution d'une saumure profonde [25]. Les diagrammes binaires montrent que plus la $\mathrm{P}_{\mathrm{CO} 2}$ augmente plus les chlorures sont mis en solution et dissolution de la Halite ( $\mathrm{Na} / \mathrm{Cl}=1)$ (Fig.5. c et d), (SWML : Current Sea Water Mixing Line)

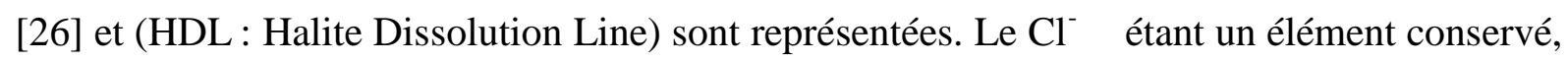
ne réagit pas avec des interactions eau-roche [27].

Les teneurs élevées en ion $\left(\mathrm{K}^{+}\right)(0.9 \mathrm{mmol} / \mathrm{L}, 0.5 \mathrm{mmol} / \mathrm{L})$ respectivement des sources $(\mathrm{S} 1)$ et (S2) et la bonne corrélation avec les chlorures indiquent (Fig.5. e) soit une dissolution de la Sylvine $(\mathrm{KCl})$ soit une hydrolyse de micas et/ou de feldspath potassique. Les faibles concentrations en $\mathrm{SO}_{4}{ }^{2-}$ de $(\mathrm{S} 2)$ et (S4) $(0.2$ et $0.08 \mathrm{mmol} / \mathrm{L})$ des eaux thermales, semblent signaler une réduction biochimique des sulfates dissous [28].

Tableau 2. Faciès géochimiques de l'ensemble des eaux analysées.

\begin{tabular}{|c|c|c|c|c|c|}
\hline Libellées & pH & $\mathbf{T}^{\circ} \mathbf{C}$ & $\begin{array}{l}\text { TDS } \\
(\mathrm{g} / \mathrm{L})\end{array}$ & $\begin{array}{c}\text { Faciès géochimiques } \\
\text { Mars-2012 }\end{array}$ & $\begin{array}{c}\text { Faciès géochimique } \\
\text { Septembre- } 2014\end{array}$ \\
\hline S1 & 6.04 & 25 & 5.2 & $\begin{array}{l}\mathrm{rHCO}_{3}^{-} \mathrm{rCl}^{-} \mathrm{rSO}_{4}{ }^{2-} \\
\mathrm{rNa}^{+} \mathrm{rCa}^{2+} \mathrm{rMg}^{2+} \mathrm{rK}^{+}\end{array}$ & $\begin{array}{l}\mathrm{rHCO}_{3}{ }^{-} \mathrm{rCl}^{-} \mathrm{rSO}_{4}{ }^{2-} \\
\mathrm{rNa}^{+} \mathrm{rCa}^{2+} \mathrm{rMg}^{2+} \mathrm{rK}^{+}\end{array}$ \\
\hline S2 & 5.93 & 39.5 & 2.4 & $\begin{array}{l}\mathrm{rHCO}_{3}{ }^{-} \mathrm{rCl}^{-} \mathrm{rSO}_{4}{ }^{2-} \\
\mathrm{rNa}^{+} \mathrm{rCa}^{2+} \mathrm{rMg}^{2+} \mathrm{rK}^{+}\end{array}$ & $\begin{array}{l}\mathrm{rHCO}_{3}{ }^{-} \mathrm{rCl}^{-} \mathrm{rSO}_{4}{ }^{2-} \\
\mathrm{rNa}^{+} \mathrm{rCa}^{2+} \mathrm{rMg}^{2+} \mathrm{rK}^{+}\end{array}$ \\
\hline S3 & 6.33 & 26 & 1.35 & $\begin{array}{l}\mathrm{rHCO}_{3}^{-} \mathrm{rCl}^{-} \mathrm{rSO}_{4}{ }^{2-} \\
\mathrm{rNa}^{+} \mathrm{rCa}^{2+} \mathrm{rMg}^{2+} \mathrm{rK}^{+}\end{array}$ & $\begin{array}{l}\mathrm{rCl}^{-} \mathrm{rHCO}_{3}^{-} \mathrm{rSO}_{4}^{2-} \mathrm{rNO}_{3} \\
\mathrm{rNa}^{+} \mathrm{rCa}^{2+} \mathrm{rMg}^{2+} \mathrm{rK}^{+}\end{array}$ \\
\hline S4 & 6.34 & 39 & 1.3 & $\begin{array}{l}\mathrm{rHCO}_{3}{ }^{-} \mathrm{rCl}^{-} \mathrm{rSO}_{4}{ }^{2-} \\
\mathrm{rNa}^{+} \mathrm{rCa}^{2+} \mathrm{rMg}^{2+} \mathrm{rK}^{+}\end{array}$ & $\begin{array}{l}\mathrm{rHCO}_{3}{ }^{-} \mathrm{rCl}^{-} \mathrm{rSO}_{4}{ }^{2-} \\
\mathrm{rNa}^{+} \mathrm{rCa}^{2+} \mathrm{rMg}^{2+} \mathrm{rK}^{+}\end{array}$ \\
\hline $\mathrm{D} / \mathrm{D} 4$ & 6.25 & 20.6 & 1 & $\begin{array}{l}\mathrm{rHCO}_{3}^{-} \mathrm{rCl}^{-} \mathrm{rSO}_{4}{ }^{2-} \mathrm{rNO}_{3}^{-} \\
\mathrm{rCa}^{2+} \mathrm{rNa}^{+} \mathrm{rMg}^{2+} \mathrm{rK}^{+}\end{array}$ & $\begin{array}{l}\mathrm{rHCO}_{3}^{-} \mathrm{rSO}_{4}^{2-} \mathrm{rCl}^{-} \quad \mathrm{rNO}_{3}^{-} \\
\mathrm{rCa}^{2+} \mathrm{rNa}^{+} \mathrm{rMg}^{2+} \mathrm{rK}^{+}\end{array}$ \\
\hline $\mathrm{D} / \mathrm{MS} 1$ & 6.33 & 17.4 & 0.6 & $\begin{array}{l}\mathrm{rHCO}_{3}^{-} \mathrm{rCl}^{-} \mathrm{rNO}_{3}^{-} \mathrm{rSO}_{4}^{2-} \\
\mathrm{rCa}^{2+} \mathrm{rNa}^{+} \mathrm{rMg}^{2+} \mathrm{rK}^{+}\end{array}$ & $\mathrm{Nm}$ \\
\hline
\end{tabular}

Les paramètres physiques sont ceux de la période Mars- 2012. 
Nm: Non mesuré en cette période.
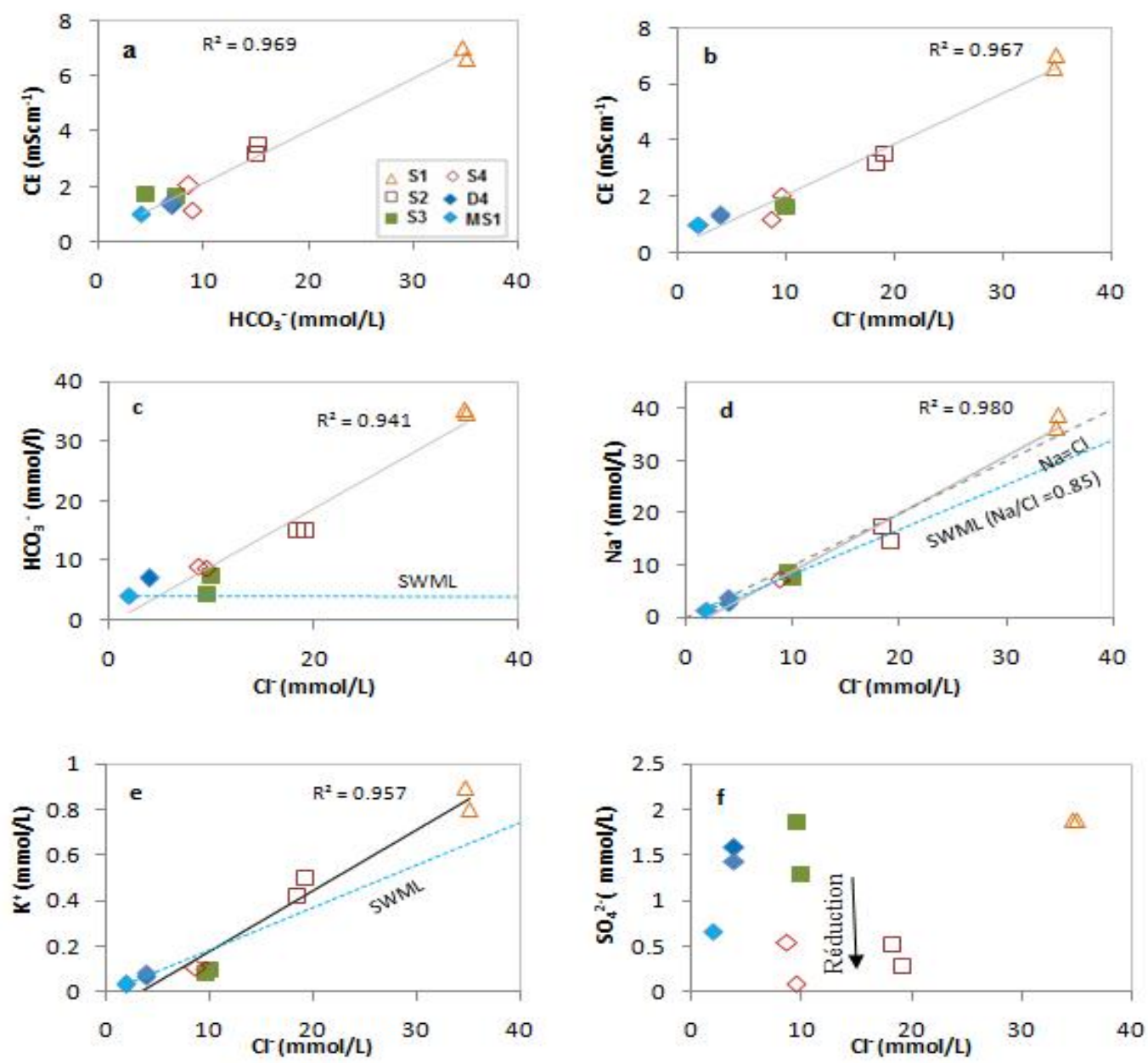

Fig. 5. Corrélations entre $\mathrm{CE}$ et $\mathrm{HCO}_{3}{ }^{-}, \mathrm{CE}$ et $\mathrm{Cl}^{-}$. $\mathrm{HCO}_{3}{ }^{-}, \mathrm{Na}^{+}, \mathrm{K}^{+}$et $\mathrm{SO}_{4}{ }^{2-}$ et les concentrations en $\mathrm{Cl}^{-}$.

\section{3. Éléments traces}

Les éléments traces utilisés sont $\left(\mathrm{Sr}^{2+}, \mathrm{F}^{-}\right.$et $\left.\mathrm{Br}^{-}\right)$, des eaux thermales des groupes (I et II) ont des concentrations notables en strontium particulièrement l'eau (S1) (0.87mmol/L), indiquant soit une dissolution de Célestine $\left(\mathrm{SrSO}_{4}\right)$ et/ou de Strontianite $\left(\mathrm{SrCO}_{3}\right)$. Celle de (S2) $(0.12 \mathrm{mmol} / \mathrm{L})$ suggère un contact et une dissolution de minéraux évaporitiques, et /ou contribution d'une saumure profonde à l'écoulement (Fig. 6. b), les eaux froides du groupe (III) contiennent des teneurs insignifiantes en strontium $(0.008 \mathrm{mmol} / \mathrm{L})$, l'eau de (S3) présente une teneur intermédiaire de $(0.03 \mathrm{mmol} / \mathrm{L})$. Les concentrations en fluor des eaux thermales sont élevées (supérieurs à $0.12 \mathrm{mmol} / \mathrm{L}$ ), celles des eaux froides sont très faibles 
(0.01mmol/L). Dans la relation $\mathrm{F}^{-}$et $\mathrm{SiO}_{2}$, le Fluor aurait une origine profonde. Plus la $\mathrm{T}^{\circ} \mathrm{C}$ augmente plus les silicates sont mis en solution graphe (Fig. 6. a et c). Les teneurs élevées de l'élément fluorure des eaux thermales pouvant témoigner de la participation d'eau profonde. Afin de déterminer l'origine des chlorures [29] sont reportés en fonction des concentrations en $\mathrm{Cl}^{-}$le rapport moyen $\mathrm{Br} / \mathrm{Cl}$ de l'eau de mer $\left(1.53 \pm 0.01 \times 10^{-3}\right)$ [30] et celui de l'Halite $\left(0.1 \times 10^{-3}\right)$ [25] (Fig. 6. d), les eaux froides se trouvent nettement au dessus de la droite de l'eau de mer, celles des eaux thermalesse trouvent en dessous, les eaux chaudes sont comprises entre la droite de l'Halite et la droite de l'eau de mer, ce qui indique l'influence de la Halite.

L'odeur de soufre dégagée par les eaux (S1), (S2) et (S4) témoigne de la présence du sulfure d'hydrogène endogène; pour [31] les eaux de $(\mathrm{S} 2)$ et $(\mathrm{S} 4)$ sont sulfureuses. Le $\left(\mathrm{H}_{2} \mathrm{~S}\right)$ présent dans les eaux thermales est le résultat d'une réduction biochimique des sulfates dissous. L'émergence (S1) avant nos travaux, était une source méconnus.
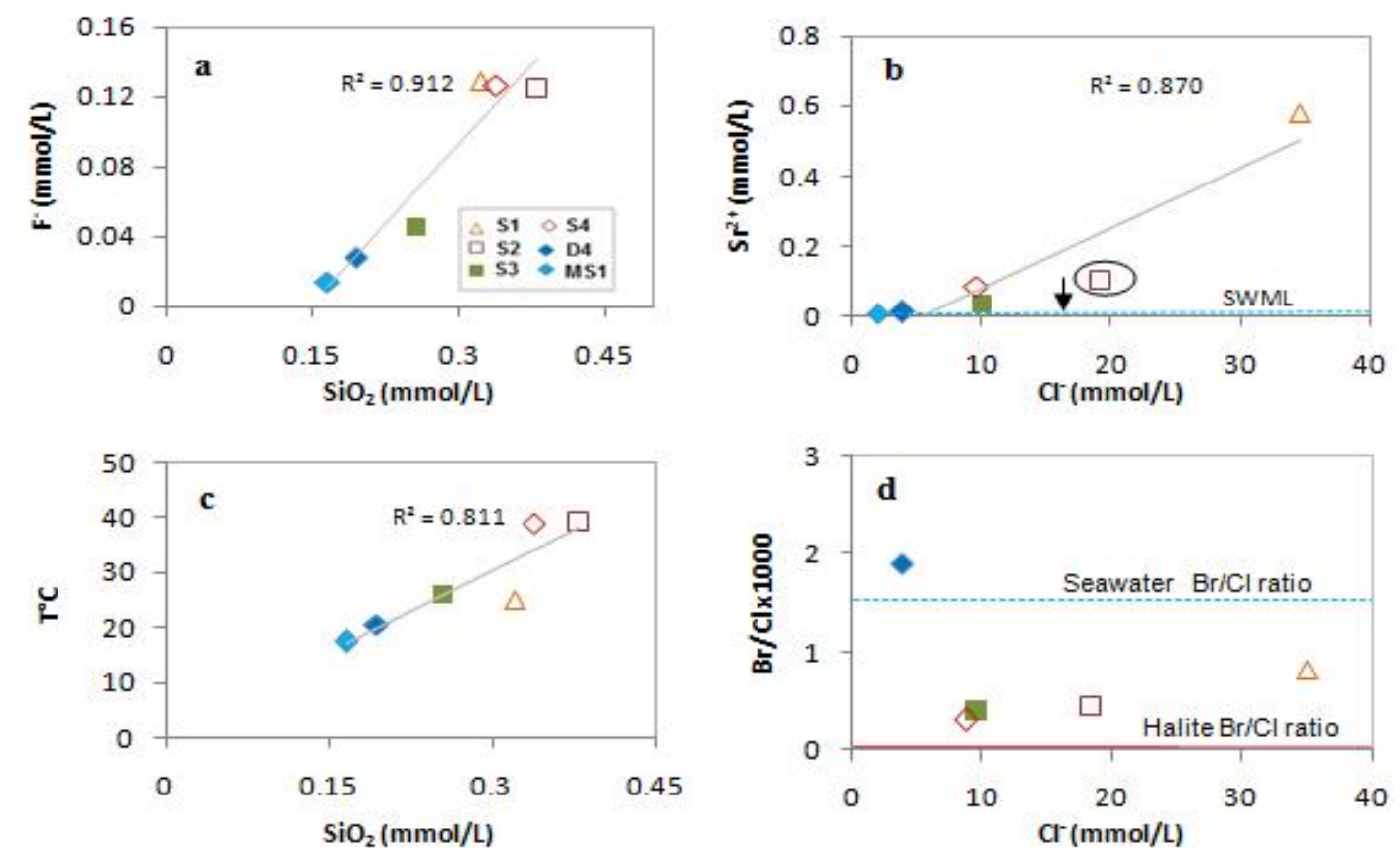

Fig. 6. Corrélations du $\mathrm{F}^{-}$et $\mathrm{T}^{\circ}$ avec $\mathrm{SiO}_{2}$. $\quad \mathrm{Sr}^{2+}$ et $\mathrm{Br} / \mathrm{Cl}$ avec $\mathrm{Cl}^{-}$.

\subsection{Variables du système calco-catbonique et du gypse}

L'état de saturation du complexe aquifère en période de basses eaux (septembre 2014), montre des $\mathrm{P}_{\mathrm{CO} 2}$ particulièrement élevées pour les sources thermales (S1) et (S2) Table 3. Les importants apports de $\mathrm{CO}_{2}$ sont d'origine profonde. Ils conduisent à affirmer qu'actuellement 
les eaux qui circulent dans l'aquifère carbonaté possèdent un fort potentiel de dissolution de la zone noyée du karst. Cette dissolution met en place une karstification particulière se développant du bas vers le haut, le long des accidents distensifs majeurs favorisant à la fois la diffusion des gaz profonds et la remontée des eaux thermales. En surface, les travertins à proximité des sources thermales $(\mathrm{S} 1)(\mathrm{S} 2)$ et $(\mathrm{S} 3)$ témoignent en partie de cette dissolution. En effet, la nette sursaturation en calcite des eaux (S1) exprime un dégazage du $\mathrm{CO}_{2}$ avant émergence et un début de précipitation du minéral.

La nette sous saturation en dolomite et les faibles valeurs du rapport molaire $\mathrm{Mg} / \mathrm{Ca}$ calculées pour la majorité des eaux s'expliquent par une quantité importante de $\mathrm{CO}_{2}$ aqueux $\left(\mathrm{H}_{2} \mathrm{CO}_{3}\right)$ agissant sur les conditions d'équilibre de l'eau. Ceci traduit un dégazage de $\mathrm{CO}_{2}$ et formation de sel de calcium $\mathrm{CaCO}_{3}$; cet état de fait induit des circulations d'eau en milieu aéré généré par la karstification des fractures distensives.

L'indice de saturation vis-à-vis du gypse très négatif pour toutes les eaux et les faibles concentrations en sulfates, semblent confirmer une réduction des sulfates par les microorganismes.

Tableau 3. Variables du système calco-carbonique et du gypse des prélèvements d'eau

\begin{tabular}{lccccc}
\hline \multicolumn{1}{c}{ Libellés } & $\begin{array}{c}\mathbf{P}_{\mathbf{C O 2}} \\
\mathbf{( A t m )}\end{array}$ & $\mathbf{I S c}$ & $\mathbf{I S d}$ & $\mathbf{I S g}$ & $\mathbf{M g} / \mathbf{C a}$ \\
\hline S1 (Mars-2012) & 1.778 & 0.33 & 0.35 & -1.09 & 0.36 \\
S2 (Mars- 2012) & 1.348 & 0.02 & -0.54 & -1.85 & 0.14 \\
S3 (Mars-2012) & 0.229 & -0.25 & -1.05 & -1.31 & 0.21 \\
S4 (Mars-2012) & 0.316 & -0.1 & -0.37 & -2.6 & 0.38 \\
D/D4 (Mars-2012) & 0.251 & -0.4 & -1.36 & -1.18 & 0.23 \\
D/MS1 (Mars-2012) & 0.12 & -0.72 & -2.38 & -1.61 & 0.11 \\
S1 (Septembre -2014) & 3.548 & -0.2 & -0.47 & -1.16 & 0.60 \\
S2 (Septembre-2014) & 2.089 & -0.4 & -1.3 & -1.64 & 0.17 \\
S3 (Septembre-2014) & 0.039 & -0.19 & -0.81 & -1.25 & 0.27 \\
S4 (Septembre-2014) & 0.331 & -0.25 & -0.75 & -1.72 & 0.32 \\
D/D4 (Septembre-2014) & 0.223 & -0.58 & -1.58 & -1.26 & 0.32 \\
\hline
\end{tabular}

\section{CONCLUSION}

L'hydrogéochimie du complexe aquifère du bassin de Souk Ahras indique divers types de circulation d'eaux à différents niveaux: eau de recharge transitant par le karst, interactions 
eau- roche à faible température, interactions eau- roche à forte température avec mise en solution de minéraux du Trias. Le couple teneurs en fluor et silice montre que les eaux chaudes sont issues et/ou parcourent de niveaux très profonds. Les teneurs élevés en potassium des eaux thermales des sources $(\mathrm{S} 1)$ et $(\mathrm{S} 2)$ indiquent une mise en solution de minéraux riches en potassium provenant du Trias et/ou probablement du socle paléozoïque. Les valeurs anormales calculées de $\mathrm{P}_{\mathrm{CO} 2}$ indiquent une origine très profonde du gaz carbonique. Le dispositif structural; failles en extension, l'émergence de la principale source thermale à proximité d'un fossé d'effondrement et le diapirisme à partir de failles profondes, attestent une contribution d'un fluide très profond salin mêlé aux écoulements d'eau jusqu'à l'émergence, perceptible tout particulière au niveau de la source (S2).

\section{ACKNOWLEDGEMENTS}

Les auteurs remercient : l'Agence Nationale des Ressources Hydrauliques (ANRH), le Centre de Recherche Nucléaire d'Alger et le laboratoire GIS de Nîmes pour les analyses des eaux.

\section{REFERENCES}

[1] Ferraga A. Ressources en eaux des karsts du Nord Est Algérien. Mémoire de Thèse de docteur- Ingénieur, Hydrogéologie. Université des Sciences et Techniques du Languedoc de Montpellier, France, 1985. 166p.

[2] ANRH. Energoprojekt. Réalisation de la Carte des Ressources en Eau Souterraine du Nord de l'Algérie, 2009. http://www.anrh.dz.

[3] ANRH. Etude de modélisation de la nappe de Rémila- Khenchela, 2004. Progress, 206p. Rapport Interne.

[4] Djebbar M. Le système karstique hydrothermal constantinois (Algérie nord orientale): structure et fonctionnement. Acte 8ème colloque d'hydrogéologie en pays calcaire, AISH, UNESCO, Neuchâtel., 2006. 89 - 92.

[5] Bouroubi Y. Étude hydrogéologique du synclinal de la Taoura. Fonctionnement et évaluation des ressources en eau souterraine. Magister, Université de Constantine, 2009. $184 \mathrm{p}$. 
[6] Boudoukha A, Athemna M. Journal of Water Science. 2, 2012, 103-118, doi: 10.7202/1011602ar.

[7] Kedaid F Z. Data base on the geothermal resources of Algeria. Revue Geothermics., 2007, 36 (3), 265-275.

[8] Saibi H. Geothermal resources in Algeria. Renewable and Sustainable Energy Reviews., 2009, 13 (5), 2544-2552.

[9] Vila J M. La Chaîne alpine d'Algérie orientale et des confins algéro-tunisiens. Thèse Docteur ès Sciences, 1980. Université. P et M Curie. Paris VI. 450p.

[10] ANRH. Carte Pluviométrique de l'Algérie du Nord au 1/500000, 1993. www.anrh.dz/cartes.htm.

[11] ABH. Agence de Bassin hydrographique. Le bassin versant de la Medjarda- Mellegue.

Les Cahiers de l'Agence, 2005. Journal n9. 28p.

[12] Durand Delga M. Mise au point sur la structure du Nord-Est de la Berbérie, Publ. Serv.

Géol. Algérie, 39, 1969, 89-131.

[13] David L. Etude géologique des monts de la haute Medjerda. Thèse Sci. Paris. Publ. Serv. Carte géol. Algérie, 1956. 304p.

[14] Bouroubi-Ouadfel Y \& Djebbar M. Etude hydrogéochimique et approche géothermique à la caractérisation du système karstique de Souk Ahras- Taoura. $1^{\text {ier }}$ Congrès international de génie civil et d'hydraulique, 10 et 11décembre 2012, Guelma-Algérie.

[15] Strojexport. Prospection géophysique du synclinal de Taoura-Bordj Mraou, 1977. Rapport Interne, Direction de l'Hydraulique de Souk Ahras.

[16] Bouroubi-Ouadfel Y, Djebbar M. Apport de la géochimie à la caractérisation des écoulements du système karstique hydrothermal du synclinal de la Taoura. $9^{\text {ième }}$ Colloque d'Hydrogéologie en Pays Calcaire ; Besançon, France, H2 Karst, 2011. pp 74-76.

[17] Haddouche O. Contribution à l'étude géologique et gîtologique des minéralisations à $\mathrm{Pb}-\mathrm{Zn}, \mathrm{Fe}(\mathrm{Ba}-\mathrm{Sr})$ d'El Ouasta (Atlas saharien oriental, Algérie). Magister, Université d'Alger, 2003.

[18] Dubourdieu G et Durozoy G. Observation tectonique dans les environs de Tébessa et de l'Ouenza; Bulletin de la Société Géologique de France. 1956. 20, (4-6), p. 257- 266. 
[19] Zouaghi T, Bédir M, Ayed-Khaled A, Lazzez M, Soua M, Amrid A, Hédi Inoubli M. Journal of Structural Geology. 52, 2013, 163-182, doi.org/10.1016/j.jsg.

[20] Yelles-Chaouche, Boudiaf A, Djellit H, Bracene R. C R. Geoscience. 338, 2006, 126-139, doi : 10.1016/j.crte.2005.11.002.

[21] Perthuisot V. Les diapirs du Maghreb oriental: part des déformations alpines et des structures initiales crétacées et éocènes dans les formes actuelles. Bulletin de la Société Géologique de France., 1999, 170 (1), 57- 65.

[22] Verdeil P. Algerian thermalism in its geostrucrural setting- How hydrogeology has helped in the elucidation of Algeria's deep seated structural. Journal of Hydrology., 1982, 56 (1-2), 107-117.

[23] Edmunds W M, Smedley P L. Applied Geochemestry. 15, 2000, 737- 752, doi: 10.1016/S0883-2927(99)00079-7.

[24] Kloppmann W. Geochimica et Cosmochimica Acta. 65, 2001, 4087-4101, doi: 10.1016/S0016-7037(01)00640-8.

[25] Fontes J Ch, Matray J M. Chemical Geology, 109, 1993, 149-175, doi: 10.1016/0009-2541(93)90068-T.

[26] Wilson T.R.S. Salinity and major elements of sea water. In P. Riley, \& Skirrow, G. (Eds.), Chemical Oceanography. New York: Academic Press, 1975, pp. 365-413.

[27] Hill D.Journal of Soil Science. 35, 1984, 27-33, doi: 10.1111/j.1365-2389.1984.tb00256.x.

[28] Canellas J, Blavoux B. Houille Blanche, 2-3, 1995, 81-86, doi: http://dx.doi.org/10.1051/lhb/1995019.

[29] Alcalá F J, Custodio E. Journal of Hydrology, 359, 2008, 189-207, doi: 10.1016/j.jhydrol.2008.06.028.

[30] Hsissou Y, Mudry J, Mania J, Bouchaoua L, Chauve P. Earth and planetary Science, 328, 1999 , 381-386, doi: 10.1016/S1251-8050(99)80103-7.

[31] Guigue S. Les sources thermominérales de l'Algérie - Étude géochimique. Bull. Serv. Carte Géol.1947. Algérie. 
How to cite this article:

Bouroubi-Ouadfel Y, Djebbar M and Khiari A. Hydrothermal complex of the SoukAhras basin: Geological and Hydrogeochemical approaches (North Est of Algeria). J. Fundam. Appl. Sci., 2016, 8(3), 894-909. 